\title{
The Digital Divide for Hispanic Americans
}

\author{
Kenneth L. Hacker \\ Department of Communication Studies, \\ New Mexico State University, \\ Las Cruces, New Mexico, USA \\ Robert Steiner \\ Experimental Statistics, Department of Economics, \\ New Mexico State University, \\ Las Cruces, New Mexico, USA
}

This study reports the data analysis of a probability sample of Anglo and Hispanic adults. The research objective guiding the study concerned discovering differences there may be in Internet usage between Anglo and Hispanic Americans. Twelve hypotheses were derived from past research and tested in this study. The findings indicate that Anglo Americans are more likely than Hispanic Americans to use the Internet and are higher on net usage frequency after becoming users. The data also show that Anglo Americans are more likely than Hispanic Americans to achieve interpersonal communication benefits from using the Internet. No significant differences were found for political communication benefits between the two groups. We also found significant ethnic differences in skills necessary for using the Internet. The process of testing these hypotheses provides more clarity of what contributes to the gaps between Hispanic and Anglo Americans in using the Internet. Overall, it appears that income, education, and ethnicity are all important to Internet usage, but in specific and differing ways.

KEYWORDS digital divide, hispanics, internet, ethnicity

$\mathbf{T}$

here is a flurry of news stories and commentaries today about the rapid growth of the Internet and the many possible uses for this new medium of communication. Internet communication is claimed to empower consumers and citizens throughout the world by providing them with new sources of information and social interaction. Notwithstanding the fact that many of these claims are hyperbolic, there is evidence that Internet users are able, in fact, to gain economic, political, financial, job-related, and

Address correspondence to Kenneth L. Hacker, Department of Communication Studies, New Mexico State University, Las Cruces, NM 88003. 


\section{DEMOGRAPHIC
VARIABLES $\Rightarrow$ HURDLES $\Rightarrow$ USAGE $\Rightarrow$ BENEFITS}

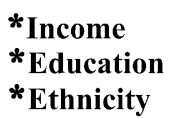

*Income

*Ethnicity

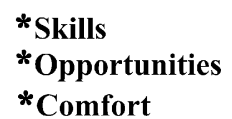

* Duration

*Frequency
* Jobs
* Financial
* Interpersonal
* Political

Figure 1 The research model.

communication benefits from using the Net (Anderson, Bikson, Law \& Mitchell, 1995; Bikson \& Panis, 1999). However, some data indicate that many minority group members are less involved with the enhanced communication that is generally attributed to the Internet (van Dijk, 2000). Worse yet, some findings indicate that some minority groups are falling further behind the majority members of American society when multiple factors of computer use and Internet usage are measured (Bikson \& Panis, 1999; Dizard, 2000; "Falling through the Net," 1999; "Falling through the Net," 2000; Paletz,1999). ${ }^{1}$

This article reports the findings of an empirical study designed to see if ethnic differences are important in Internet usage and to discover possible reasons for the existence of digital divide gaps between Hispanic and Anglo Americans in using the Internet. The study was guided by a research model that assumes that there are access or hurdles variables that must be fulfilled before benefits can be obtained by Internet use. These access ("hurdles") variables are related to three sets of demographic variables: ethnicity, income, and education. The research model is shown in Figure 1. A hurdles variable represents a condition that must be met to use the Internet and a benefits variable represents a positive outcome resulting from Internet usage.

\section{The Internet and World Wide Web}

According to the Internet survey clearinghouse Nua Internet Surveys, the United States Internet population had about 100 million users and the world user population in 1999 was approximately about 276 million users (Nua Surveys, 2000). For the year 2000, these numbers estimated by Nua increased to nearly 400 million users worldwide and about 150 million U.S. users (Nua Surveys, 2001). In 2002, Nua estimates world users at over 500 million and United States users at over 160 million. (Nua, 2002). In the U.S. in 2000, 42\% of American households had Internet access (Newburger, 2001). The latest U.S. federal government summary of Internet usage reports that by the end of 2001, over 140 million Americans were using the Internet, which is about $54 \%$ of the population ("A Nation Online," 2002). Experts generally agree that the Internet and World Wide Web have reached critical mass (Rogers \& Malhotra, 2000). A recent Internet study done by National Public Radio indicates that $75 \%$ of Americans under the age of 60 report having used the Internet ("Survey Shows Wide Enthusiasm," 2000). These facts highlight the overall rapid expansion of Internet usage but, of course, do not reveal the gaps in usage among various groups in society such as ethnic groups. 


\section{The Digital Divide Gaps Between Ethnic Groups}

Many Americans are using computer-mediated communication (GMG) such as electronic mail and the World Wide Web for locating information, discussing issues with each other, and for attempting to influence policy makers about their concerns. However, members of most ethnic minority groups in the U.S. generally have less CMC access than do Anglo citizens. Since 1995, when the RAND study on e-mail (Anderson et al. 1995) was released, studies have indicated that the gaps between ethnic groups for CMC access are large and possibly increasing. More recent RAND data indicate similar findings. According to Bikson and Panis (1999, p. 20) "We had not expected to find race/ethnicity differences in computer access and connectivity that were not attributable to variation in income, education or other demographic characteristics. The size and endurance of the differences, however, indicate that race/ethnicity gaps deserve further investigation."

The National Telecommunications and Information Administration (NTIA), documented, in 1995, 1998, 1999, and 2000 that most minority group members lag behind Anglo (majority) group members in both computer ownership and online activity ("Falling through the Net," 2000). This is true even when controlling for education and income. U.S. households with an annual income of $\$ 75,000$ or more are 20 times as likely to have Internet access as those households at the lowest income levels ("Falling through the Net," 1999). Some scholars argue that many of the digital divide gaps in Internet usage, such as ethnic gaps, have worsened in recent years (van Dijk, 2000). A recent NTIA report indicated that Internet usage gaps between Hispanic Americans and the national average increased from 1998 to 2000 from 14 to 18 percentage points. It also indicated that the gap for computer ownership between Hispanics and national average remained constant from 1998 to 2000 with the national average being 51\% and the Hispanic American average being 34\% ("Falling through the Net," 2000). Jung, Qiu, and Kim (2001) found that in a large southern California metropolitan area, 63\% of Anglo American homes have Internet access in contrast to less than $20 \%$ of Hispanic American homes. A recent U. S. Census Bureau report indicated that of children 3-17 years old in America, 38\% of Anglo children use the Internet at home whereas only $13 \%$ of Hispanic children do so (Newburger, 2001). The latest NTIA report argued that Internet usage growth for African and Hispanic Americans was increasing at an annual rate of $33 \%$ and $30 \%$, whereas the rates for Anglo and Asian Americans are only about 20\% ("A Nation Online," 2002). Within this report, however, are statistics showing that certain gaps have become worse for Internet usage. For example, the gaps between Anglo and Hispanic Americans for the years 1997, 1998, 2000, and 2001 were in succession 14\%, 21\%, 27\%, and 28\% ("A Nation Online," 2002).

If there were no tangible benefits to Internet communication, there would most likely be less concern with the digital divide, but research indicates that such benefits do exist. Katz and Aspden (1997) discovered two key motivational factors for using the Internet are personal development and social development. Personal development entails benefits such as finding desired information. Social development involves increasing abilities to communicate with others by using this new medium. High costs, difficulties in using the Internet, and having insufficient access are important barriers to using the Internet. Katz \& Aspden also found that social networks are important sources of learning how to use the Internet for new users. Numerous studies today indicate the users of the Internet are using 
it for social and work networking, seeking information, and communicating with others (Anderson et al., 1995; Castells, 2001; Hacker \& van Dijk, 2000).

\section{Uncertainties About the Digital Divide Ethnic Gaps}

Some survey researchers have challenged the claim that the digital divide is continuing as described by most of the NTIA data reports. A National Public Radio study reported, for example, that gaps between White and Black Americans in Internet use declined with levels of income ("Survey Shows Widespread Enthusiasm," 2000). Earlier research by Hoffman and Novak (1998) indicated that Anglo Americans are more likely to report that they have used the World Wide Web at home than are African Americans. They also found that differences between Whites and Blacks diminish at higher income levels, but not with education levels (Hoffman \& Novak, 1998). These researchers conclude that access is essential to use of the Internet and that minorities such as African Americans should be given more opportunities for net access (Hoffman \& Novak, 1998).

The NTIA surveys found that although Internet use is increasing for all ethnic groups in the United States, White Americans are more likely to have access to the net from home or any other location than are African Americans and Hispanics ("Falling through the Net," 1999). Hispanic households were found to be two-fifths as likely as Anglo households to have home Internet access. The NTIA report also indicated that gaps between Hispanic households and Anglo households has increased since 1994 ("Falling through the Net," 1999). The gaps between Anglos and African Americans narrowed for those with annual incomes over \$75,000 ("Falling through the Net," 1999). The 2000 NTIA data indicated that $24 \%$ of Hispanic Americans have Internet access at home, whereas $42 \%$ of households nationally have Internet access at home. The report also showed that the gap between Hispanics and the national percentage increased from 13.6 points in 1998 to 17.9 points in August, 2000 ("Falling Through the Net," 2000). Although the most recent NTIA report did not discuss home Internet access in detail, there are interesting facts in its tables concerning children and home access. For children 3-17 years old, 50\% Anglo, $25 \%$ of African American, and 20\% of Hispanic Amerian children have access to home computers and Internet access. For young adults between 18 and 24 years of age, the percentages are $74 \%$ for Anglo, $47 \%$ for African, and $50 \%$ for Hispanic Americans ("A Nation Online," 2002).

As seen above, there are mixed messages in various empirical studies concerning the digital divide. Some report that the ethnic gaps are narrowing; some say they are worsening. Some argue that the gaps are simply a function of income or education. It is possible that some of the mixed messages are because of the wide diversity in sampling techniques, questions asked, and how the data are summarized. Some surveys (e.g., some of those of the Pew Internet Project) measure Internet users as those who have used the Internet. This is much different than asking for the frequency of Internet usage and then using frequency as a variable. The digital divide gaps have been documented since 1995 as have the benefits that are possible from using the Internet. A great deal of uncertainty persists regarding the exact nature of the ethnic gaps and what caused or remedied these gaps. Social policy may be directly affected by this degree of uncertainty. Social scientists may be able to sort out the relationships among key variables in ways that help policy makers to make more informed decisions about lessening the ethnic gaps in the digital divide. More and more 
services are becoming available on the Internet. This suggests that leaving segments of the overall population without net access and skills or with lagging access and skills, may lock them out of or diminish their participation in the digital communication processes that comprise a growing part of the Internet communication system.

Some observers claim that Hispanic Americans will be the largest minority group by the year 2005 and will comprise about one fourth of the U.S. population by the year 2050. These claims are supported by the United States Census Bureau, which reports that the Hispanic percentage of the United States population in 2002 is $12 \%$ and will grow to $24.3 \%$ by 2050 and $26 \%$ by the year $2055 .^{2}$ This simple observation makes it compelling to ascertain why there are gaps between Hispanic and Anglo net users, particularly when many researchers find that income and education do not explain the differences.

Some organizations, mainly political or marketing in nature, have suggested that income explains the divide between ethnic groups and Internet use. Yet, a Tomas Riveras Institute study found that at every income group below $\$ 75,000$, there are substantial differences between Hispanics and non-Hispanics ("Buying into the Computer Age...," 1998). When socioeconomic factors like income and education are held constant, Hispanics continue to remain less likely than non-Hispanics to own computers. Hispanic parents appear to get some of their evaluations of computers from the opinions of their children using computers in schools. They are also concerned about the non-computer aspects of education including bilingual teaching and learning materials so they can help their children with homework ("Buying into the Computer Age...," 1998). There is less of a perceived need for computer-assisted learning if it is not bilingual. Additionally, if the children receive help with computers at school, there is less perceived need to have computers at home. While some value the computer as something that would be good to buy, others prioritize taking care of basic needs higher than buying a computer. Perceived advantages of computer ownership include making certain activities like banking more convenient, accessing information, and enhancing one's chances of getting good jobs. Perceived disadvantages include children using the Internet for pornography, children playing games rather than doing homework with computers, and replacing time spent with family members by time spent alone at a computer. Many express concerns about high costs of access, time invested to learn, and difficulty in learning the new technologies. Many also indicate interests in taking classes to learn about computers to lessen feelings of apprehension about computers ("Buying into the Computer Age...," 1998).

van Dijk (2000) described categories of CMC and Internet usage upon which we based our research model (Figure 1). van Dijk listed four hurdles of access to people benefiting from Internet use: (a) lack of basic skills and computer fear, (b) low access to computer networks, (c) insufficient user-friendliness, and(d) insufficient and unevenly distributed usage opportunities. The van Dijk model is consistent with the research findings of Katz \& Aspden (1997) described earlier. It can also be related to Uses and Gratifications Theory which says that people use communication technologies to accomplish specific ends. According to this theory, the communication technology user chooses particular media for specific needs and knows how to meet certain needs by such choices (Littlejohn, 1999).

A review of literature about the digital divide reveals that much of the controversy regarding the digital divide is founded on isolated findings rather than upon rigorous hypothesis testing. The hypotheses tested in this study represent four major areas of focus: demographics (income, education, ethnicity), hurdles or access variables, usage frequency, and benefits variables. The access variable of skills refers to subjects' self-reported 
level of skill in using the Internet. The comfort variable is their self-reported ease in using the Internet. The opportunities access variable is their self-reported ability to use the Internet when they wish to use it. Benefits variables include perceived job, financial, interpersonal communication, and political benefits obtained from using the Internet.

\section{Hypotheses}

\section{Hurdles and Ethnicity}

Because of the differences in reported data about Anglos having higher Internet usage than Hispanics, it is reasonable to expect that Anglo Americans have had more success in meeting the hurdles of Internet access. Anglo Americans were higher in home computer adoption in the mid-1990s ("Falling through the Net," 1995) and are still higher today in Internet usage and home Internet access ("Falling through the Net," 2001; "A Nation Online," 2002). Previous work indicates that users must meet certain hurdles to gain access and then usage (van Dijk, 1999). We therefore hypothesize that Anglo Americans are advantaged over Hispanic Americans in meeting these hurdles in that:

Hypothesis 1: Anglos will perceive having higher Internet skills than Hispanics.

Hypothesis 2: Anglos will perceive having higher Internet opportunities than Hispanics.

Hypothesis 3: Anglos will perceive having higher Internet comfort than Hispanics.

\section{Comparative Hypotheses for Income, Education, and Ethnicity}

Some studies, while acknowledging ethnic gaps, argue that income is more important and that as income increases, ethnic gaps in Internet usage decrease ("A Nation Online," 2002; "Falling through the Net," 1999; "Survey Shows Widespread Enthusiasm," 2000). If income explains some of the variance that is attributable to ethnicity, it is possible that income differences are more explanatory concerning gaps in the digital divide.

Hypothesis 4: Income is more highly related than ethnicity to Internet usage frequency.

Although income and ethnicity may provide some barriers to Internet usage frequency, educational settings offer computer labs and other points of access that should encourage usage. Classes and educational activities also promote the use of computers and computer networks. For those without home access, educational settings can provide the missed access opportunities (Hoffman \& Novak, 1998; Horrigan \& Rainie, 2002).

Hypothesis 5: Education is more highly related to Internet usage frequency than is ethnicity.

Ethnicity has been shown in various reports to have an effect that is not completely or sufficiently accounted for by higher levels of education or income (Bikson \& Panis, 1999; Castells, 2001; "Falling through the Net," 1999; Hoffman \& Novak, 1998; Katz \& Aspden, 1997). Therefore, ethnicity is likely to have differential effects on the hurdles variables for Internet usage frequency even when controlling for income and education. For those who use the Internet, we expect: 
Hypothesis 6: Controlling for both income and education, Anglos are higher on Internet skills.

Hypothesis 7: Controlling for both income and education, Anglos are higher on Internet usage opportunities.

Hypothesis 8: Controlling for both income and education, Anglos are higher on comfort with Internet usage.

As with the hurdles that need to be met for Internet communication and the reports indicating ethnic differences despite control for income and education, it is reasonable to expect that ethnic differences will be significant for Internet usage benefits. Studies that have controlled for income and education have not been able to explain away the independent effects of ethnic group membership despite the fact that some of the effect of ethnicity diminishes at the higher income (over \$75,000 annual income) levels (Bikson \& Panis, 1999; Castells, 2001; "Falling Through the Net," 2000).

Hypothesis 9: Controlling for both income and education, Anglos are higher on the job benefits acquired from using the Internet.

Hypothesis 10: Controlling for both income and education, Anglos are higher on the financial benefits acquired from using the Internet.

Hypothesis 11: Controlling for both income and education, Anglos are higher on the interpersonal communication benefits acquired from using the Internet.

Hypothesis 12: Controlling for both income and education, Anglos are higher on the political information benefits acquired from using the Internet.

\section{Methodology}

Respondents of this study were sampled from the municipal area in the Southwest. The total population of this area is approximately 176,000 . The area is known for having a strong Hispanic cultural component.

A random sample telephone survey was used to gather data. Telephone numbers were generated at random. This included stratification for prefix areas within the calling district. A sample of 319 surveys was generated in a 3-day period. The response rate for this sample was calculated at $.38 .^{3}$

Eleven trained phone interviewers were used to randomly call members of the population. One interviewer was fluent in Spanish and was given those participants who could not speak English but spoke Spanish. We had a substantial opening statement that introduced the survey, however, we did not ask for language preference in our introduction. If the person indicated they did not speak English, we switched to a Spanish-speaking interviewer. Also, if an interviewee began speaking Spanish, we had the Spanish speaker shift to the Spanish introduction and Spanish questions.

A survey was administered to address each research question. Likert-type scales were used to gain responses to questions concerning sections of our research model such as hurdles, usage, and benefits of usage. Survey questions asked respondents about how they meet the hurdles (access variables) of Internet usage and what benefits they receive from using the Internet. Hurdles questions concerned issues like having enough skills, access, 
and opportunities to use the Internet. Benefits variables are perceived job, financial, political, and interpersonal benefits derived from using the Internet.

For each hurdle and benefit concern, three items were asked. Those that were reliable enough to combine into indices were then used for the analysis. This procedure resulted in three hurdle indices and four benefits indices. Individual items (with Alpha values) comprising the indices are listed in the appendix. Reliability for the benefit variables was tested by having three items for each benefit. All of the items were correlated sufficiently to allow the creation of four benefits indices. For the jobs index, the Cronbach alpha for the individual items was .90. The Cronbach alpha for the finance index was .93. For the interpersonal communication index, the Cronbach alpha was .96. For the political information index, the Cronbach alpha was .97. Hypotheses were tested by $t$ tests, correlation, and analysis of variance.

\section{Results}

The probability sampling produced a participant pool that is 55\% Anglo, 36\% Hispanic, $1 \%$ Asian, and the rest African American, Native American, and others. ${ }^{4}$ Regarding education, $19 \%$ of our sample has an education level of either high school education or below and $16 \%$ of the respondents have a graduate degree. For income, $24 \%$ have an income level less than $\$ 20,000$ and $19 \%$ have an income of over $\$ 60,000$. For gender, $42 \%$ of the sample is male and $58 \%$ is female. For age, $38 \%$ of the participants are under $35,29 \%$ are between 35 and 49 , and $33 \%$ are over 50 years of age.

In this study, we have two subsets of participants, one consisting of Internet users $(n=139)$ and the other of non-users $(n=117)$. As we look at differences between the user set and the non-user set, we find that differences in education and income are greatest between these two subsets of our overall sample. That is, for the Internet users, that sample is rather homogeneous on education and income levels whereas the total sample of users and non-users shows heterogeneity on these variables for the two ethnic groups. Despite this important observation, however, we focus mainly on the Internet users for data concerning access and benefits variables since we are looking for alleged ethnic differences in Internet access and Internet benefits.

Table 1 compares Anglo Americans' use of the Internet with that of Hispanics. ${ }^{5} \mathrm{~A} \chi^{2}$ test indicates that there are significant differences, $\mathrm{X}^{2}=4.05, d f=1, p<.05$, in their use. However, we found in comparing three levels of income (low, medium, high) to whether or not a person uses the Internet that are there insignificant differences $\mathrm{X}^{2}=2.483, d f=2, p>.05$. This is true despite the fact that in the highest income category, $72 \%$ of our participants are Internet users in contrast to $56 \%$ for medium income and $53 \%$ for low income. There are no reversed directions in the income levels; each level has more users than non-users.

\section{Table 1 Ethnicity and Using or Not Using the Internet}

\begin{tabular}{lcc}
\hline Internet use & Anglos & Hispanics \\
\hline Using the Internet & 59.35 & 46.53 \\
Not using the Internet & 40.65 & 53.47 \\
\hline
\end{tabular}

Note. Chi-square $=4.502(d f=1)$. Significant at $p<.05$. 
Table 2 Education and Using or Not Using the Internet

\begin{tabular}{lccc}
\hline Internet use & Low & Medium & High \\
\hline Using the Internet & 23.64 & 60.56 & 70.91 \\
Not using the Internet & 76.36 & 39.44 & 29.09 \\
\hline
\end{tabular}

Note. Chi-square $=29.23(d f=2)$. Significant at $p<.001$.

A different picture emerged for education and whether or not a person uses the Internet. Here we found that there are significant differences for levels (low, medium, high) of education $\left(\mathrm{X}^{2}=29.23, d f=2, p<.0001\right)$. There is also an interesting reversal in direction for levels of education and Internet use, namely that for medium and high education, there are many more users than non-users; but for low education, $76 \%$ are non-users. These data can be seen in Table 2 .

\section{Usage, Hurdles, and Benefits}

Before testing for ethnic group differences, it was necessary to test the basic assumptions of our research model that links hurdles of access to frequency of usage and usage frequency to benefits.

Our data indicate that for all of the participants who are using the Internet, Internet usage frequency is significantly correlated with success in meeting the skills $(r=.40$, $p<.01)$ and opportunities $(r=.48, p<.01)$ and comfort $(r=.33, p<.01)$ hurdles. We also found that all four types of benefits (jobs, financial, interpersonal communication, political) are significantly correlated with net usage frequency (see Table 3). However, we need to note that the correlations, although statistically significant, are low.

A Spearman correlation analysis indicates that the skills hurdle is significantly but not highly correlated with all four benefits: job $(r=.34, p<.001)$, financial $(r=.18$, $p<.05)$, interpersonal $(r=.34, p<.001)$, and political $(\mathrm{r}=.25, p<.01)$. The opportunities hurdle is significantly correlated with the job benefit variable $(r=.24, p<.01)$, the interpersonal communication variable $(r=.36, p<.001)$, and the political benefit variable $(r=.31, p<.001)$, but not with the financial benefit variable $(r=.14, p>.05)$. The comfort hurdle index is significantly correlated with job benefits $(r=.36, p<.001)$. It is significantly correlated with financial benefits $(r=.21, p<.05)$, interpersonal benefits $(r=.24$, $p<.01)$, and political benefits $(r=.25, p>.01)$.

Table 3 Benefits by Usage Spearman Correlation Coefficients

\begin{tabular}{lcccc}
\hline Usage & Job & Financial & Interpersonal & Political \\
\hline Correlation & .28 & .27 & .34 & .33 \\
$p$ value & $.0014^{* *}$ & $.0018^{* *}$ & $.0001^{* *}$ & $.0002^{* *}$ \\
$n$ & 123 & 123 & 126 & 124 \\
\hline
\end{tabular}

**significant at $p<.01$. 


\section{Hurdles and Ethnicity}

As shown in Table 4, Anglos report having more Internet skills than Hispanics $(p<.05)$. We also found evidence that there are greater Anglo opportunities for using the Internet. As shown in Table 4, the difference between Anglos and Hispanics on comfort using the Internet is not significant $(p>.05)$. Thus, our first two hypotheses were confirmed and the third was not. We must note, however, that some of the means were statistically significant but the practical difference is questionable. ${ }^{6}$

\section{Hurdles, Income, and Education}

We examined our data to see how income and education are correlated with hurdles of using the Internet. Income and overcoming the skills hurdle are not significantly correlated $(r=-.08, p>.05)$. There is a statistically significant correlation of income and overcoming the opportunities hurdles $(r=.18, p<.05)$. Despite being statistically significant, however, the correlation is very small. There is not a significant correlation between income and overcoming the comfort hurdle $(r=-.03, p>.05)$. In fact, our data indicate that there is no significant correlation between education and any of the three hurdles indices.

\section{Internet Usage Benefits and Ethnicity}

We found that most Internet benefit index means (see Table 5) do not differ significantly between Anglos and Hispanics. Differences in job benefits derived from using the Internet for the two ethnic groups are not significant $(t=1.05, p>.05)$. Financial benefits

\section{Table 4 Hurdles and Ethnicity Tables}

\begin{tabular}{lccr}
\hline Hurdle & $n$ & $M$ & $S D$ \\
\hline Skills Hurdle by Ethnicity & & & \\
$\quad$ Skills & 91 & 11.52 & 2.77 \\
$\quad$ Anglos & 47 & 10.57 & 2.72 \\
$\quad$ Hispanics & & & \\
$\quad t=.1 .90, p<.05$ & & & \\
Opportunities Hurdle by Ethnicity & & 11.53 & 2.48 \\
$\quad$ Opportunities & 90 & 10.49 & \\
$\quad$ Anglos & 47 & & \\
$\quad$ Hispanics & & & \\
$\quad t=1.93, p<.05$ & & 12.00 & 2.29 \\
Comfort Hurdle by Ethnicity & & 11.72 & \\
$\quad$ Comfort & 90 & & \\
$\quad$ Anglos & 47 & & \\
$\quad$ Hispanics & & & \\
$\quad t=0.70, p>.05$ & & & \\
\hline
\end{tabular}




\section{Table 5 Benefits by Ethnicity}

\begin{tabular}{lccr}
\hline Benefit & $n$ & $M$ & $S D$ \\
\hline Job benefits & & & \\
Anglos & 86 & 8.57 & 3.85 \\
$\quad$ Hispanics & 46 & 7.85 & 3.65 \\
Financial & & & 3.77 \\
Anglos & 90 & 8.04 & 3.21 \\
$\quad$ Hispanics & 47 & 7.04 & 3.54 \\
Interpersonal & & & 3.74 \\
Anglos & 89 & 9.11 & 4.19 \\
Hispanics & 47 & & 4.22 \\
Political & & 7.99 & \\
Anglos & 85 & & \\
Hispanics & 47 & & \\
\hline
\end{tabular}

$*=$ significant at $p<.05$.

differences are also insignificant $(t=1.55, p>.05)$. Political benefits are not significantly different among the two groups $(t=1.30, p>.05)$. However, interpersonal communication benefits are significantly higher for Anglos than for Hispanics $(t=3.08, p<.01)$.

Our analysis thus far has shown us the following about ethnic group differences and Internet communication. First, we found that ethnic differences are significant for Internet skills and opportunities but not for comfort in using the Net. Second, we found that income is not significantly related to skills and comfort while income is significantly related to opportunities to use the Internet. We should note that this last relationship, while statistically significant, is a weak with a correlation of only.18. Third, we found that job, financial, and political benefits are not significantly different for the two ethnic groups, while interpersonal communication benefits are significantly different.

\section{Accounting for Education and Income: Hypotheses 4-12}

Concerning those who use the Internet, analysis of variance (ANOVA) indicates the following regarding the Hypotheses 4-12. Looking at income and ethnicity together as independent variables and Internet usage frequency as a dependent variable, ethnicity is significant as an independent variable $(F=5.02, d f=1, p<.05)$ and income is not $(F=0.32, d f=2, p>.05)$. When testing income as an independent variable and usage frequency as the dependent variable, we find that income is not significant as a predictor in an ANOVA analysis $(F=0.51, p>.05)$. An ANOVA test of ethnicity on usage frequency, however, shows that ethnicity is significant as the independent variable $(F=5.49, p<.05)$. This refutes the hypothesis that income is more highly related than ethnicity to Internet usage frequency. The same pattern is found for education and ethnicity. Ethnicity significantly predicts Internet usage frequency $(F=5.3, d f=1, p<.05)$, whereas education is not significant as the independent variable $(F=0.22, p>.05)$. In an ANOVA analysis, education is not a significant predictor of usage frequency $(F=0.26, p>.05)$. This refutes the 
hypothesis that education is more highly related to Internet usage frequency than is ethnicity.

Looking at the hurdles or access variables with ANOVA tests, we found the following. For the skills variable, looking at ethnicity and education as independent variables and skills as the dependent variable, ethnicity is significant $(F=3.00, p<.05)$. For opportunities, ethnicity and education are both insignificant predictors. There are no significant relationships in the ANOVA involving ethnicity and education as independent variables and the comfort access variable $(F=0.56, d f=3, p>.05)$ as the dependent variable. ${ }^{7}$ Neither of the two independent variables is a significant predictor. Income is a significant predictor of skills $(F=3.49, p<.05)$ as is ethnicity $(F=4.08, p<.05)$, but education $(F=2.83, p>.05)$ is not. From these tests, it appears that when both income and education are controlled, Anglos are higher on Internet skills (Hypothesis 6).

Neither education, income or ethnicity alone predict the opportunities index. A test for opportunities as the dependent variable shows that neither education, income, or ethnicity is a significant predictor. For the comfort access or hurdles variable, we see the same pattern as found for the opportunities variable. Education, income, and ethnicity are insignificant predictors of comfort using the Internet. These results appear to refute Hypotheses 7 and 8, which states that, controlling for both income and education, Anglos are higher on Internet usage opportunities and on comfort with Internet usage.

For the benefits variables and last hypotheses, we treated ethnicity, income, and education as independent variables and the four benefits variables as dependent variables in ANOVA tests. The results show that none of the independent variables predict job benefits or financial benefits. These results appear to refute Hypotheses 9 and 10. For interpersonal communication benefits, the data indicate that ethnicity is significantly associated with gaining interpersonal communication benefits from using the Internet $(F=8.83, p<.01)$, thereby confirming Hypothesis 11. For the political benefits dependent variable, no significant effects were found for education, income, or ethnicity. This refutes Hypothesis 12.

\section{Discussion}

In summary, we found the following about Anglo and Hispanic American differences in Internet usage with our testing of specific hypotheses. First, we found that Internet usage frequency is correlated significantly for all users with skills, opportunities, and comfort. We also found that for all users we sampled, Internet usage frequency is significantly related to obtaining job, financial, interpersonal communication, and political communication benefits. Next, we found that there are digital divide gaps between Hispanic and Anglo Americans in Internet skills and opportunities for using the Internet with Anglo Americans having the advantage.

We also found that income and the skills and comfort hurdles are not significantly related but that income and the hurdles variable of opportunities are significantly related. For education, we found that education is not significantly related to any of the hurdles variables (skills, opportunities, comfort). We discovered that combining income, education, and ethnicity as predictor variables in models predicting Internet usage, success with hurdles (skills, opportunities, comfort), and gaining benefits from Internet usage (job, financial, interpersonal, political) account for low amounts of variance. 
In terms of specific hypotheses, we confirmed that Anglos have higher self-reported skills and opportunities than Hispanic Internet users, but refuted the hypothesis of greater comfort for the Anglo Americans. We refuted the hypothesis that income is more related than ethnicity to usage frequency. We also refuted the hypothesis that education is more important than ethnicity for usage frequency. We found support for the hypothesis that Anglos are higher on skills when controlling for education and income. We refuted the hypothesis that Anglos are higher on opportunities when controlling for education and income. The same is true for the hypothesis that Anglos are higher on comfort when controlling for education and income. Similarly, the hypotheses about higher Anglo job benefits, financial benefits, and political benefits were refuted. However, the hypothesis that Anglos are higher on interpersonal communication benefits when controlling for education and income was confirmed.

\section{Conclusions}

It appears that there are some significant gaps between Anglo and Hispanic American net users in our sample and these gaps are significantly related to the hurdles of skills in using the Internet and opportunities for usage of the Internet. Also, we found no evidence that income and/or education negate or subsume ethnic differences in Internet usage frequency or benefits.

The low correlations among hurdles and usage frequency and between usage frequency and benefits indicate that, although significant, the associations between the variables are not strong ones. While we find specific disconfirmation or confirmation for hypotheses about ethnic gaps, we have little data that indicate why these gaps exist. More research is clearly needed to identify these reasons for the gaps.

Rupertus and Straubhaar (2001) examined publications aimed at minority and Anglo groups and found that advertising for computer technology was more prevalent for Anglos than for Hispanic and African Americans. Other sources have argued cultural reasons for ethnic group differences in the digital divide. We earlier mentioned the Tomas Rivera Policy Institute's claims about concerns regarding pornography and taking away time from family communication. The Benton Foundation argued that some recent marketing research indicates that Hispanic Americans are feeling more pressure to keep up and some of the pressure comes from their children who wish to be skilled in using computers (Becht, Taglang, \& Wilhelm, 1999). They also said there are findings indicating that Hispanics like using the Internet to connect to nations of their heritage as well as cultural sites (Becht et al., 1999).

Castells (2001) disagreed with a cultural explanation for ethnic gaps in the digital divide. He argued that the ethnic gaps are understood in relation to how access for various groups change over time. Castells noted, as does the latest NTIA report, that the rates of growth are higher for Hispanics than for Anglo Americans. However, he also noted that the gaps between the two groups have increased over time (Castells, 2001). Castells argued that once income and education are held constant, Hispanic Americans are less likely than Anglo American to have home computers and also less likely to have opportunities outside of the home to use computers (Castells, 2001). He said that these two facts contribute to less Internet access. Because none of his arguments contradict the data reported by the Tomas Rivera Policy Institute and Benton Foundation researchers, it 
may be that Castells and these other researchers all have valid possible reasons for the ethnic gaps.

If we wish to take an empirical approach to making conclusions about the ethnic gaps in the digital divide, we are presently stuck with the observations that the gaps are real and we still do not have anything approaching consensus about why they exist. However, we do have data from various sources indicating possible cultural reasons, reasons to some extent related to income and education, and also differences in types of access and usage opportunities. Clearly, more research is needed to sort out the determinants of the ethnic group differences in the digital divide.

Some of the findings in this study confirm previous work on the ethnic digital divide gaps and other findings do not. Despite the variations in numbers regarding the ethnic aspects of the digital divide, it remains clear that Hispanic Americans have not had the same rate of Internet adoption as Anglo Americans. This suggests that there may be an ethnic lag in what are referred to as network society and digital democracy. However, this lag has something to do with ethnicity that is not yet explained.

As we have argued, more work is needed to link what we know about the importance of ethnicity and communication in general to the study of the digital divide. This work may profit by examining what we know about ethnic differences in communication in general and differences in how ethnic groups organize themselves in society. Cornell and Hartmann (1998) observed that ethnic identities are used by ethnic group members to organize their daily lives and the more marginalized their group is from the total society, the more the salient is the group identity. What is known as "enclave theory" suggests that ethnic groups who are disadvantaged in the economy often tend to form intra-ethnic networks of communication and commerce (Cornell \& Hartmann, 1998, p. 63). If this has any connection to the digital divide, it might be in the possibility that because Hispanic Americans have suffered discrimination and marginalization that Anglo Americans have not, they have formed tighter ethnic community ties that make Internet communication less pressing as a need. If Hispanic Americans are finding more Hispanic and Spanishlanguage sites of interest, it may also be a possibility that their rate of adoption will continue to increase.

By identifying what hurdles and benefits are most important for these ethnic differences, policy makers can make more informed decisions about what to do about the digital divide. This study has made a modest step in that direction. Although our probability sample is limited to one geographic area of the American population, it allowed us to test various detailed hypotheses about hurdles and benefits in relation to ethnic differences. National-scale probability samples can replicate our testing with much greater external validity. Already we can see important consistencies among the NTIA and Pew national sample data and our study. First, we see that ethnic gaps persist yet close for the older $\mathrm{S}$ curves (as in the case of basic Internet access) and open again for new $S$ curves (as in the case of broadband Internet service at home). We also see that ethnic gaps are greatest at the point of access hurdles and diminish at the points of usage and benefits.

Although it can be argued that gaps between Hispanic Americans and Anglo Americans have been lessening with time, the gaps are more complex than simply having physical connections to a computer linked to the Internet. It has been correctly argued that there is a classic $\mathrm{S}$ curve of adoption for each new communication technology (Rogers, 1986). Yet, it is crucial to realize that Internet adoption differs from television and radio adoption for two basic reasons. First, using computers and the Internet requires active 
skills that are simply nonexistent in the passive process of attending TVand radio. Second, Internet usage adoption is cumulative; that is, one technology release builds on the previous one. It begins with computer adoption and skills and continues through network skills, software usage skills, programming skills, search-and-retrieval skills, and so on. It also includes the varying layers of equipment sophistication both in terms of PCs and in terms of network connection bandwidth. As the ethnic gaps are slowly improving on Internet usage in general, there is a whole new $S$ curve with new gaps for broadband Internet connections from homes. A Recent Pew Internet Project report indicates that $85 \%$ of broadband net users are Anglo Americans, whereas only 5\% are Hispanic Americans (Horrigan \& Rainie, 2002). This is important for many reasons including the fact that broadband users are doing things that dial-up users are not. The broadband users are more active at creating content such as Web sites, posting opinions, sharing files, and information retrieval (Horrigan \& Rainie, 2002).

The facilitation of digital democracy for all Americans may require efforts in communication that brings more analysis of empirical data concerning what factors are related to use and what factors of access and benefits vary among ethnic groups. We have possible cultural and access reasons that need to be included in better research models. For example, education may affect income and income may have an effect on access variables at the same time as ethnicity independently affects access. The digital divide is probably not as simple as many studies have made it seem and better communication research can illuminate why the gaps exist and how they create differences in communication practices and benefits for various ethnic groups.

\section{Notes}

\footnotetext{
${ }^{1}$ One ethnic group that does not generally suffer substantial gaps from the Anglo American group is what is known as the Asian American group. Asian Americans generally appear to have higher Internet access than all other groups.

${ }^{2}$ These Census Bureau statistics are available at http://www.census.gov.

${ }^{3}$ Although this is not an optimal response for a survey, methods experts note that pollsters doing national random-sample surveys claim response success in the 25-40\% range (Frey, Botan \& Kreps, 2000).

${ }^{4}$ We followed the recommendations of the American Anthropological Association and did not use the term race because of its historical baggage and questionable validity, instead using the term ethnicity and allowing participants to self-identify their ethnic group membership.

${ }^{5}$ This seems to be the common and uncomfortable problem of differences that are statistically significant but not of clear practical difference.

${ }^{6}$ When we refer to use of the Internet, this is using or not using the Internet as opposed to our variable "Internet usage frequency," which is a measure of amount of use.

${ }^{7}$ When we tested a model of income, education, and ethnicity together as independent variables, including two-way interactions with the skills variable as the dependent variables, we find that the overall model is significant $(F=2.10, d f=13, p<.05)$.
}

\section{References}

Anderson, R. H., Bikson, T. K., Law, S. A., \& Mitchell, B. M. (1995). Universal access to e-mail: Feasibility and societal implications. Santa Monica: Rand.

Becht, D., Taglang, K. , \& Wilhelm, A. (1999). The digital divide and the U.S. Hispanic population. Digital Beat, 1(13), 6. Retrieved from http://www.benton.org/DigitalBeat/db080699.html\#trpc

Bikson, T. K., \& Panis, G. W. A. (1999). Citizens, computers, and connectivity. Santa Monica, CA: Rand.

Buying into the computer age: A look at Hispanic families. (1998). Claremont, CA: Tomas Rivera Institute. 
Castells, M. (2001). The Internet galaxy. New York: Oxford University Press.

Cornell, S. and Hartmann, D. (1998). Ethnicity and Race. London: Pine Forge Press.

Davis, R. (1999). The web of politics. New York: Oxford University Press.

Davis, R., \& Owen, D. (1998). New media and American politics. New York: Oxford University Press.

Digital Divide Basics Fact Sheet. (2002). Retrieved from http://www.digitaldividenetwork.org/ content/stories/index.cfm?key $=168$

Dizard, W. (2000). Old media new media. New York: Longman.

Falling through the net: A survey of the "have nots" in rural and urban America. (1995). Retrieved from http:// www.ntia.doc.gov/ntiahome/fallingthru.html

Falling through the Net:Defining the digital divide. (1999). Retrieved from http://www.ntia.doc.gov/ ntiahome/fttn99/contents.html

Falling through the net: Toward digital inclusion.: (2000). Retrieved from http://www.ntia.doc.gov/ ntiahome/fttn00/contents00.html

Falling through the net II: New data on the digital divide. (1998). Retrieved from http://www.ntia.doc.gov/ ntiahome/net2http://www.ntia.doc.gov/ntiahome/fallingthru.html

Frey, L. R., Botan, C. H., \& Kreps, G. L. (2000). Investigating communication: An introduction to research methods. Boston: Allyn and Bacon.

Hacker, K., \& van Dijk, J. (Eds.) (2000). Digital democracy: Issues of theory and practice. London: Sage.

Hoffman, D., \& Novak, T. (1998). Bridging the digital divide on the Internet. Science, pp. 390-391.

Horrigan, J., \& Rainie, L. (2002). The broadband difference: How online Americans'behaviorchanges with high-speed Internet connections at home. Retrieved from http://www.pewinternet.org

Jung, J., Qiu, J., \& Kim, Y. (2001). Internet connectedness and inequality: Beyond the "divide." Communication Research, 28(9), 507-535.

Katz, J., \& Aspden, P. (1997). Motivations for and barriers to Internet usage: Results of a National Public Opinion Survey. Internet Research, 7, 170-188.

Littlejohn, S. (1999). Theories of human communication (6th ed.). Belmont, CA: Wadsworth.

Matei, S., \& Ball-Rokeach, S. J. (2001). Real and virtual social ties: Connections in the everyday lives of seven ethnic neighborhoods. Paper presented to the Communication and Technology division of the International Communication Association. Washington, D.C. May, 2001.

A nation online: Internet use in America. (2002). Retrieved from http://www.ntia.doc.gov/ opadhome/digitalnation/index.html\#Previous

Newburger, E. (2001). Home computers and Internet use in the United States. Washington, DC: United States Census Bureau.

Nua Surveys. (2000). How many online? Retrieved from http://www.nua.ie/surveys/ how many_online/ index.html

Nua Surveys. (2001). How many online? Retrieved from http://www.nua.ie/surveys/ how many_online/ world.html

Paletz, D. (1999). The media in American politics. New York: Longman.

Pavlik, V. J. (1998). New media technology. Boston: Allyn \& Bacon.

Rogers, E. M. (1986). Communication technology. New York: Free Press.

Rogers, E., \& Malhotra (2000). Computers as communication: The rise of digital democracy. In K. Hacker \& J. van Dijk, (Eds.), Digital democracy: Issues oft theory and practice. (pp. 10-29). London: Sage.

Rupertus, J., \& Staubhaar, J. (2001, May). This Internet is NOT for you: On not marketing the Internet to minorities. Paper presented to the Communication and Technology Division of the International Communication Association, Washington, DC.

van Dijk, J. (1999). The network society. London: Sage.

van Dijk, J. (2000). Widening information gaps and policies of prevention. In K. Hacker \& J. van Dijk (Eds.), Digital democracy: Issues of theory and practice. (pp. 166-183). London: Sage. 


\section{Appendix}

\section{Items and Indices for Hurdles and Benefits}

Skills

- I have the skills to use the Internet.

- I am skilled at using the Internet.

- My Internet skills are good.

Cronbach Coefficient $\alpha=.89$

Opportunities

- It is possible for me to use the Internet a lot.

- I am able to use the Internet when I want to.

- I have many opportunities to use the Internet.

Cronbach Coefficient $\alpha=.79$

Comfort

- I feel nervous about using the Internet.

- The Internet is comfortable for me to use.

- I do not feel comfortable using the Internet.

Gronbach Coefficient $\alpha=.74$ 
Copyright of Howard Journal of Communications is the property of Taylor \& Francis Ltd and its content may not be copied or emailed to multiple sites or posted to a listserv without the copyright holder's express written permission. However, users may print, download, or email articles for individual use. 